\title{
Refinement of diagnosis and supporting evidence for the use of immunotherapy through sequential biopsies in a case of EML4-ALK positive lung
}

\section{cancer}

This article was published in the following Dove Press journal:

OncoTargets and Therapy

\section{Peng Song' \\ Jingcheng Zhang ${ }^{2}$ \\ Li Zhangl \\ 'Department of Respiratory Medicine, Peking Union Medical College Hospital, Chinese Academy of Medical Science \& Peking Union Medical College, Beijing, People's Republic of China; ${ }^{2}$ Department of Internal Medicine, Chinese Academy of Medical Science \& Peking Union Medical College, Beijing, People's Republic of China}

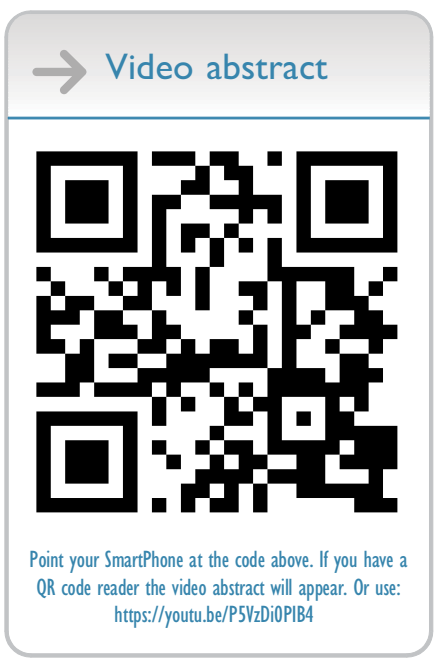

Correspondence: Li Zhang Department of Respiratory Medicine, Peking Union Medical College Hospital, Chinese Academy of Medical Science \& Peking Union Medical College, Xinkai Road, Dongcheng, Beijing, People's Republic of China

Email zhanglipumch@aliyun.com

\begin{abstract}
In this case report, we describe a tortuous, yet rare, treatment process of the patient. The first biopsy of the patient suggested inflammatory myofibroblastic tumor, ALK (D5F3) positive. Considering the benign progression of the disease, and no indication for surgical resection, oral prednisone was given first. However, the disease rapidly progressed, and a second biopsy revealed a pulmonary sarcomatoid cancer. Since the biopsy was ALK (D5F3) positive, the effect of crizotinib treatment was significant, though crizotinib resistance unfortunately only occurred after 4 months. The third biopsy pathology was performed and confirmed lung adenocarcinoma. After switching to pembrolizumab treatment, the lesions were significantly reduced after four courses of treatment. The current condition of patient persisted in partial response.
\end{abstract}

Keywords: sequential biopsies, EML4-ALK, immunotherapy, lung adenocarcinoma

\section{Case report}

A 49-year-old Chinese male suffered with cough and shortness of breath. A chest CT scan indicated a right lower lobe mass combined with the enlargement of multiple lymph nodes at mediastinal and right hilar. No abnormalities were found in either the head MRI or bone PET-CT scan. Bronchoscopy examination revealed that the mass of basal segment of the right lower lobe occluded the trachea, and the pathology suggested inflammatory myofibroblastic tumor (IMT) (Figure 1A). Immunohistochemistry (IHC) staining revealed the following: TTF-1(+), P43(+), ALK(D5F3) (+) (Figure 1B). We suggested taking oral prednisone in the first month at $50 \mathrm{mg}$ per day, then reducing the dose in the following 20 days at $30 \mathrm{mg}$ per day. Fifty days after taking prednisone, the patient experienced wheezing and difficulty breathing. Chest CT scan showed there were multiple metastatic nodules of the lungs and right lower lobe obstructive pneumonia with atelectasis (Figure 2A-C). Accordingly, a second bronchoscopy examination was conducted, revealing that the trachea was completely blocked by the mass of basal segment of the right lower lobe. Biopsy was performed and pathology showed a malignant spindle cell tumor, which could be considered as pulmonary sarcoma-like carcinoma (PSC) (Figure 1C). IHC: TTF-1(+), CK7(-), Ki-67(index about 10\%), SMA $(+)$. A second-generation gene sequencing revealed EML4-ALK fusion 

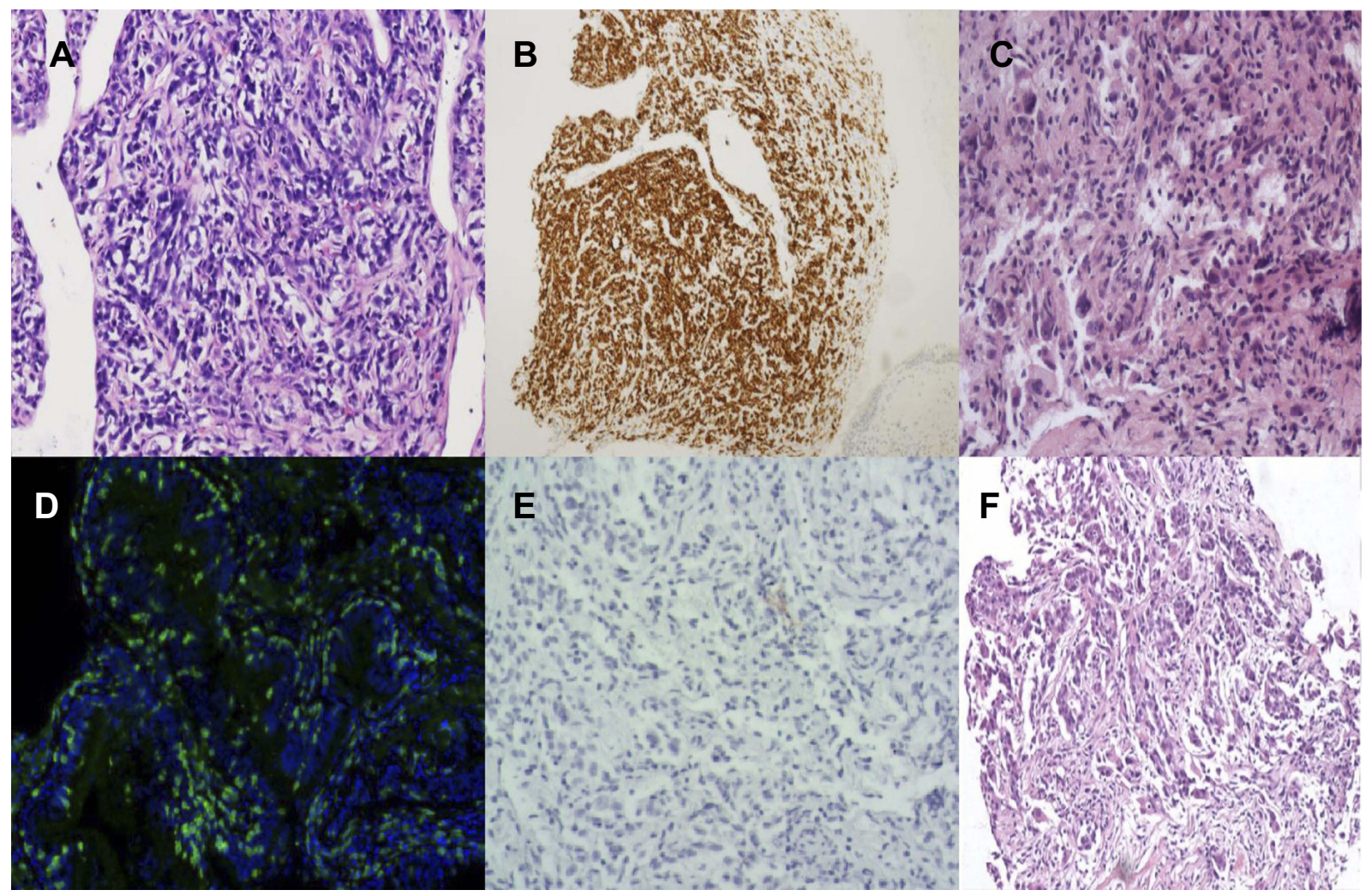

Figure I Three biopsy pathological diagnosis results of the patient.

(Figure 3A and B). Tumor mutation burden was $11.6 \mathrm{Mbs} /$ $\mathrm{MB}$ and PD-L1 detection was negative (Figure 1D). Immunocytoplasmic detection showed that $\mathrm{CD} 8+\mathrm{T}$ cells accounted for $9.07 \%$ of the total number of nuclear cells (Figure 1E). Considering the poor effect of prednisone treatment, we switched to oral crizotinib at $250 \mathrm{mg}$ twice a day. After taking crizotinib for 8 weeks, re-examination of chest CT scan showed the enlarged mediastinal lymph nodes shrank, and right lung obstructive pneumonia was significantly better than before. We assessed the condition as partial response (Figure 2D-F). After taking crizotinib for 4 months, a re-examination of chest CT suggested that mediastinal and hilar lymph nodes were larger than before, and right lung obstructive pneumonia increased more than before, showing partial atelectasis (Figure 2G-I). We discontinued the crizotinib and did a third biopsy to clarify the mechanism of drug resistance. The pathology confirmed lung adenocarcinoma (Figure 1F). IHC: TTF-1 $(+$ ), CK-7(+), P40(+). The tumor tissue second-generation gene sequencing revealed EML4-ALK fusion and $A L K-L 1196 M$ mutation (Figure 3C). Tumor mutation burden was 10.4 $\mathrm{Mbs} / \mathrm{MB}$. However, Ceritinib was not approved in China. According to the history of the patient - which had a specific manifestation of multiple tumor components, high tumor mutation burden (TMB) values, and high-density infiltration of CD8 + T cells - we issued pembrolizumab to the patient (200 mg pembrolizumab was dissolved in $0.9 \%$ normal saline $100 \mathrm{~mL}$ ), and the intravenous infusion was completed within half an hour. The intravenous infusion was given one time every 21 days. After two cycles of pembrolizumab, the chest and abdomen CT showed an increase and fusion of mediastinal lymph nodes (Figure 2J-L), but the patient reported significant improvement in physical strength. Considering the pseudo-progression of the lung lesions, the use of pembrolizumab was continued. After four cycles of pembrolizumab, the patient's self-reported physical strength and appetite improved significantly, and there was no obvious discomfort. With an ECOG of 0 points, the chest CT showed the mediastinal lymph nodes were significantly reduced (Figure 2M-O), which were assessed as a partial response. The patient engaged in continuous follow-up until then.

\section{Discussion}

IMT is a low-grade malignant tumor. Most IMTs have benign behaviors while they can also be localized invasion $(<5 \%)^{1}$. The lung is the most common organ affected by 


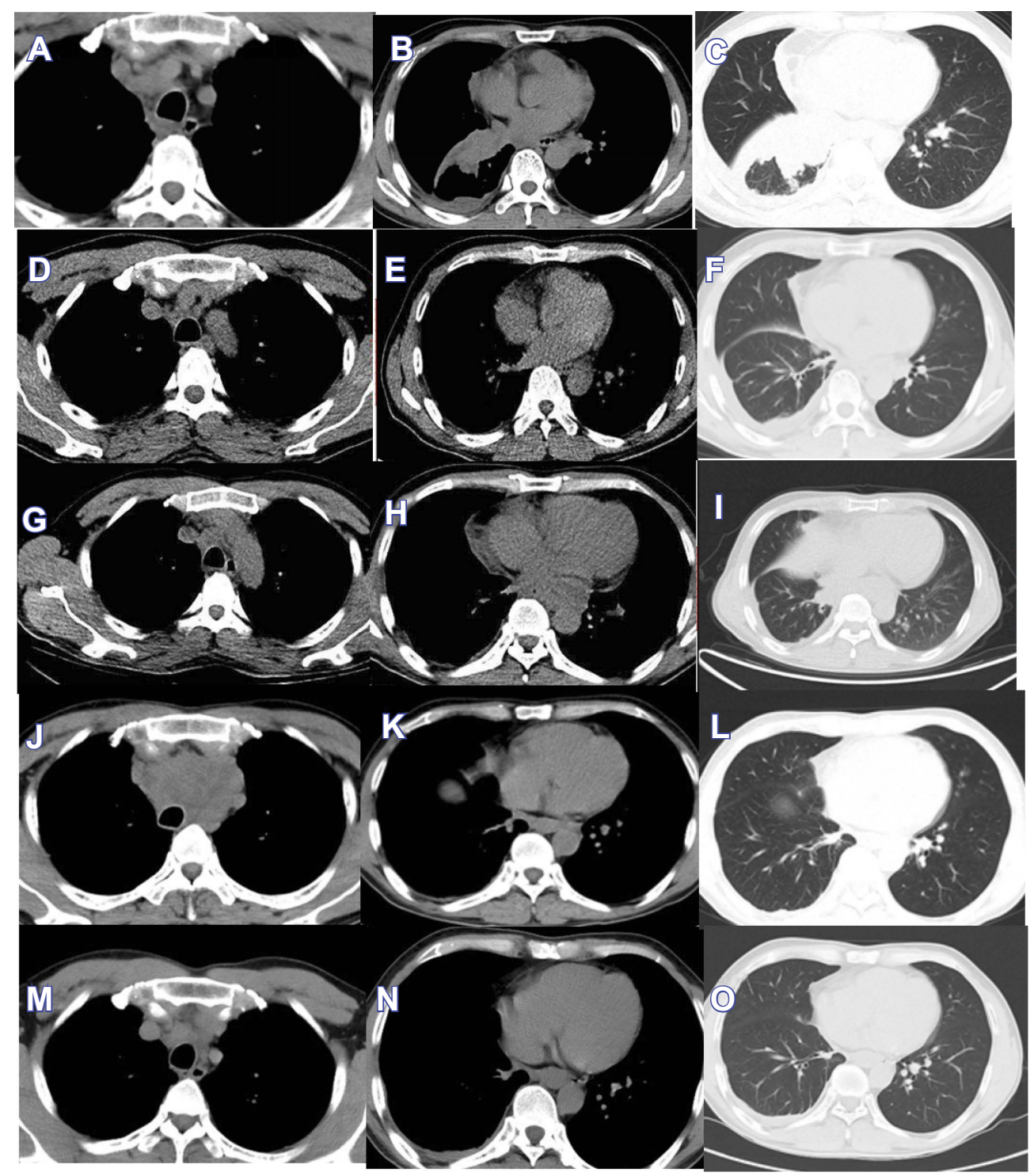

Figure 2 Chest CT scan evolution of the tumors.

IMTs, and the incidence rate is about $0.04-0.7 \%$. IMT is characterized by the mixing of myofibroblastic spindle cells with hyaluronic stroma and varying degrees of inflammatory infiltration. Coffin $\mathrm{CM}$ et al found that about $44-61 \%$ of IMTs showed ALK fusion gene rearrangement, ${ }^{2}$ and lung IMT had a higher positive ratio of ALK expression. At present, it is difficult to treat the disease for those patients who are unable to meet the surgical requirements since hormone and chemotherapy efficacy is unclear. Hiroyuki Mano named this kind of tumor "ALKoma", which carries the abnormal ALK fusion gene as an essential growth driver and shares the activated ALK gene. ${ }^{3} \mathrm{Wu}$ et al followed up the efficacy of crizotinib in nine patients with ALK-positive IMT. The objective response rate was $66.7 \%$, showing good clinical efficacy for ALK inhibitors. ${ }^{4}$ This patient had a significant response after oral administration of crizotinib, which is consistent with the "ALKoma" theory. However, his PFS was only 3 months. After gene sequencing of the drugresistant lung puncture biopsy, an ALK L1196M mutation was found, which is the resistance mechanism of crizotinib-related ALK kinase region mutation. ${ }^{4}$ There are no reports on the use of PD-1 monoclonal antibody in IMT treatment, and it is expected that PD-1 monoclonal antibody can become the future therapeutic direction of IMT.

PSC is a rare subtype of non-small cell lung cancer (NSCLC), accounting for approximately $0.4 \%$ of all lung malignancies. ${ }^{5}$ Histologically, PSC is often characterized by the mixing of conventional components of NSCLC with sarcomatoid components or heterologous sarcoma 


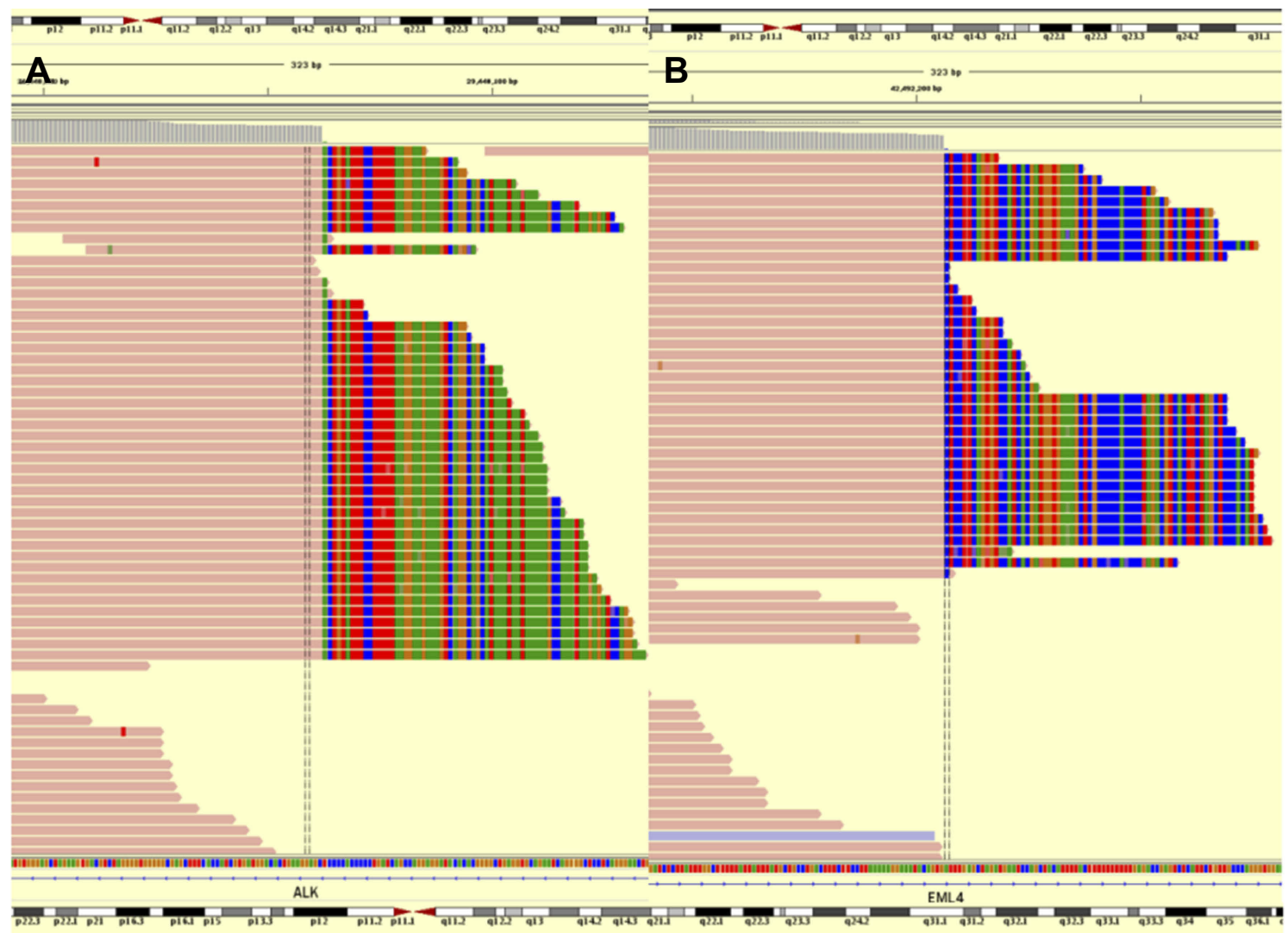

\begin{tabular}{|c|c|c|c|c|c|c|}
\hline $\mathbf{C}^{\text {sonsenon }}$ & meosenos & moosens & meosem & mensen" & menesen & mensen \\
\hline
\end{tabular}

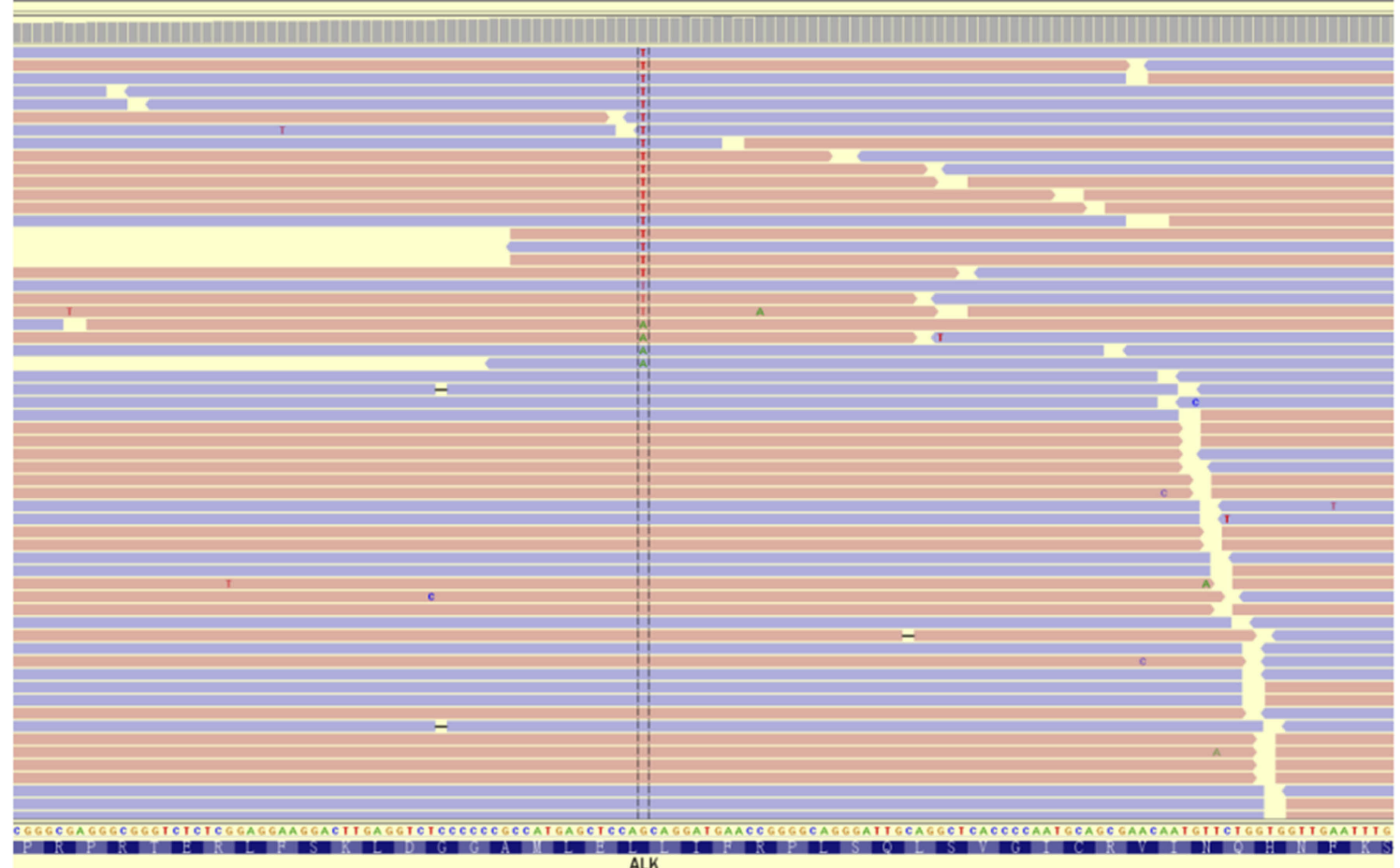

Figure 3 IGV screenshots (EML4-ALK) of the fusion sample. 
tissue. TP53 is the most common mutated gene of PSC, followed by $K R A S$ and PIK3CA, with the ALK fusion gene having also been reported. ${ }^{6}$ Greta Alì et al reported the first double primary tumors case that had positive EML4-ALK rearrangement, confirming the lung sarcomatoid carcinoma and lung adenocarcinoma by pathology. ${ }^{7}$ Patients with PSC are not sensitive to conventional radiotherapy, which may be associated with the high frequency of $K R A S$ mutations, leading to a difficulty in treatment and poor prognosis. Filippo Lococo et al found that the TMB value in PSC patients was quite high, and the use of immunotherapy might be a new possibility for treatment. ${ }^{6}$

Three large multi-center international trials - CheckMate 057, KEYNOTE-010, and POPLAR - have demonstrated that PD-1/PD-L1 inhibitors in patients with EGFR mutations or ALK fusion are less effective than wild-type patients. ${ }^{8-10}$ The recently published ATLANTIC study found that patients with ALK-rearranged NSCLC had a higher frequency of PD-L1 overexpression than patients with EGFR-mutant NSCLC, though Duvalumab was only observed to be effective in EGFR-mutant NSCLC patients, suggesting that they had differing immunobiology. ${ }^{11}$ It is currently accepted that immunological checkpoint inhibitors are not effective in patients with ALK-rearranged NSCLC. However, in this case, the ALK-positive patient was treated twice with immunological checkpoint inhibitors (keytruda), and the lesions were significantly reduced, which was considered to be associated with high TMB and abundant CD8 $+\mathrm{T}$ cell infiltration. This result suggests that clinicians should assess TMB and immune microenvironment in patients with targeted drug-resistant ALK-positive malignancies.

Since there was no surgical indication in this patient, the three pathology specimens were small specimen biopsies of the primary tumor. Therefore, there was not a sufficient amount of tissues to evaluate the clonal relationship between the three tumors by array comparative genomic hybridization. By identifying changes in the genome copy numbers, this method can determine whether the three tumors are multiple primary tumors. Moreover, three different pathological findings in the same site showed that the patient's tumor was highly heterogeneous, suggesting that clinicians should use gene mutation detection and immune microenvironment detection for such patients. The precise tumor treatment plan (basket plan), which is based on molecular pathology rather than the malignancy of traditional pathological types, will be the future direction of development. The first clinical trial of the basket plan, NCI-MATCH, is still underway, and it is expected that the results of the trial will be announced soon. ${ }^{12}$

\section{Conclusion}

When the treatment of malignant lung tumors is not effective, a second biopsy, or even multiple biopsies, should be performed in time. Further, the gene mutation detection and immune microenvironment detection should also be performed. From our experience, PD-1/ PD-L1 mAb may be the best choice for high heterogeneous tumor tissues.

\section{Informed consent}

Written informed consent had been provided by the patient to have the case details and any accompanying images published. This was an observational case report and institutional approval was not required, because all treatment received by this patient is standard therapy, including the crizotinib and pembrolizumab.

\section{Disclosure}

The authors report no conflicts of interest in this work.

\section{References}

1. Al-Shibli KI, Donnem T, Al-Saad S, Persson M, Bremnes RM, Busund L-T. Prognostic effect of epithelial and stromal lymphocyte infiltration in non-small cell lung cancer. Clin Cancer Res. 2008;14:5220. doi:10.1158/1078-0432.CCR-08-0133

2. Coffin CM, Hornick JL, Fletcher CD. Inflammatory myofi- broblastic tumor: comparison of clinicopathologic, histologic, and immunohistochemical features including ALK expression in atypical and aggressive cases. Am J Surg Pathol. 2007;31(4):509-520. doi:10.1097/01. pas.0000213393.57322.c7

3. Mano H. ALKoma: a cancer subtype with a shared target. Cancer Discov. 2012;2(6):495-502. doi:10.1158/2159-8290.CD-12-0009

4. Wu J, Savooji J, Liu D. Second- and third-generation ALK inhibitors for non-small cell lung cancer. $J$ Hematol Oncol. 2016;9:19. doi:10.1186/s13045-016-0251-8

5. Yendamuri S, Caty L, Pine M, et al. Outcomes of sarcomatoid carcinoma of the lung: a surveillance, epidemiology, and end results database analysis. Surgery. 2012;152(3):397-402. doi:10.1016/j. surg.2012.05.007

6. Lococo F, Gandolfi G, Rossi G, et al. Deep sequencing analysis reveals that KRAS mutation is a marker of poor prognosis in patients with pulmonary sarcomatoid carcinoma. $J$ Thorac Oncol. 2016;11 (8):1282-1292. doi:10.1016/j.jtho.2016.04.020

7. Alì G, Proietti A, Niccoli C, et al. EML4-ALK translocation in both metachronous second primary lung sarcomatoid carcinoma and lung adenocarcinoma: a case report. Lung Cancer. 2013;81(2):297-301. doi:10.1016/j.lungcan.2013.03.016

8. Horn L, Spigel DR, Vokes EE, et al. Nivolumab versus docetaxel in previously treated patients with advanced non-small-cell lung cancer: two-year outcomes from two randomized, open-label, phase iii trials (checkmate 017 and checkmate 057). J Clin Oncol. 2017;35 (35):3924-3933. doi:10.1200/JCO.2017.74.3062 
9. Herbst RS, Baas P, Kim DW, et al. Pembrolizumab versus docetaxel for previously treated, PD-L1-positive, advanced non-small-cell lung cancer (KEYNOTE-010): a randomised controlled trial[J]. Lancet. 2016;387(10027):1540-1550. doi:10.1016/S0140-6736(15)01281-7

10. Fehrenbacher L, Spira A, Ballinger M, et al. Atezolizumab versus docetaxel for patients with previously treated non-small-cell lung can cer (POPLAR): a multicentre, open-label, phase 2 randomised contr olled trial[J]. Lancet. 2016;387(10030):1837-1846. doi:10.1016/S01406736(16)00587-0
11. Garassino MC, Cho BC, Kim JH, et al. Durvalumab as third-line or later treatment for advanced non-small-cell lung cancer (ATLANTIC): an open-label, single-arm, phase 2 study. Lancet Oncol. 2018;19(4):521-536. doi:10.1016/S1470-2045(18)30144$\mathrm{X}$

12. Conley BA, Doroshow JH. Molecular analysis for therapy choice: NCI MATCH. Semin Oncol. 2014;41(3):297-299. doi:10.1053/j. seminoncol.2014.05.002.

\section{Publish your work in this journal}

OncoTargets and Therapy is an international, peer-reviewed, open access journal focusing on the pathological basis of all cancers, potential targets for therapy and treatment protocols employed to improve the management of cancer patients. The journal also focuses on the impact of management programs and new therapeutic agents and protocols on patient perspectives such as quality of life, adherence and satisfaction. The manuscript management system is completely online and includes a very quick and fair peer-review system, which is all easy to use. Visit http://www.dovepress.com/ testimonials.php to read real quotes from published authors. 